\title{
Moving beyond DSM5 and ICD11: Acoustic Analysis for Psychological Stress on Daily-Wage Workers in India during COVID19
}

\author{
Ajay Agarwal \\ verslinfiniaudela@gmail.com \\ +916387319973 \\ Department of Computer Science \& Engineering \\ DIT University, Dehradun \\ Mussoorie Road, Makka Wala \\ Dehradun -248009
}

\begin{abstract}
Aim: To observe psychological stress in the acoustic samples of daily-wage workers belonging to different states in India

Method: The study was based on the observations obtained from prior work on detecting psychoacoustic detectors of psychological stress. Acoustic samples were obtained from interviews conducted by NDTV over a period of March-May 2020. Spectrogram and Fundamental Frequency variations were used to identify psychological stress

Results: Stark differences existed in mean fundamental frequency plots by daily-wage workers and non-stressed individuals. Distinct density variations were also observed in the acoustic samples between the two groups.

Conclusion: Psychoacoustic detectors of psychological stress were confirmed in the acoustic samples of daily wage-workers, concluding that daily-wage workers are under dire psychological stress and are susceptible to stress-related disorders.
\end{abstract}

Keywords: psychological stress, acoustic variations, daily-wage workers

\section{COVID19 and its Impact on India}

The first case of novel Coronavirus was reported in late December 2019. Since then, the SARS-nCoV19 virus has continuously been transmitted to different countries of the globe. It has claimed countless lives and infected a sense of uncertainty and fear among the individuals of the world. Unfortunately, the impact of COVID19 has been a disproportionate one for different sections of the society. While a major hit is made by the increasing transmission rate and reproductive numbers of the virulent strain, a subliminal hit is made by the increasing sense of the paranoia, anxiety, fear and uncertainty among the non-infected people that has been caused due to the existing non-pharmaceutical measures like lockdown etc. taken by the government to curb the virus. As a result, while the world combats COVID19 by guarding its frontlines by nurse, doctors and law-enforcers, the people staying at homes, looking for 
homes or even migrating towards home continue to suffer the plight that COVID19 brings on their everyday spheres of lives.

In this section, we summarize the non-biological dysfunctional impact of COVID19 on different sections of society in India. Doctors, nurses and researchers continue to fight COVID19 on the frontline by mass testing measures, admitting asymptomatic patients in hospitals and working unpaid for overtime hours to help save the infected group. Students, on the other hand, are most impacted by the temporarily shutdown of colleges, schools and universities due to ongoing lockdown measures. These students face anxiety and stressful triggers as they worry most about the completion of their opted-degree course, conduction of semester exams, conduction of national engineering/medical exams and finding alternatives for rescinding job offers and internships. Farmers are most hit by the ongoing lockdown measures as they are unable to work on their farms and transport their harvest to the local markets for purpose of sale. Their savings continue to diminish and vicious debt traps continue to worry them. Employees working from homes are in constant stress and anxiety over their paychecks. By the current norms ensued by the government, companies can only open with one-third of their employees, the employees fear stiff competition for their chance of securing their position in their offices. They fear that they are likely to be fired or terminated from their jobs, without getting pay of the preceding months of lockdown. Tenants fear that their landlords are going to demand exorbitant rents even during such crisis times, in lieu of not asking them to vacate their homes. Finally, the section of population most hit by COVID19 are the migrant workers, or daily-wage workers. Comprising a significant part of the workforce in India, they fear the lack of employment opportunities post-COVID19. As their survival is extremely dependent on the daily-wage they receive at the end of their daily turmoil, the absence of the same (wages) haunts them and forces them to migrate to their home towns and villages in search of their families and money for survival. Many NGOs, influential celebrities and government institutions have provided aid to them to return safely to their villages and home towns. [2-6].

\section{Triggers of Stress during Public Crisis}

Psychological stress is a comorbidity with public crisis events. Over the years, various public crisis events have occurred across the world. These events range from terrorism, pandemics, natural calamities or extremism. The occurrence of such events is followed by event-specific measures to curb their future occurrence. As a result, a psychological ripple of stressful triggers in generated among the individuals who have either been first-hand witness to such events, first-hand survivors, or even people remotely associated by/effected by the measures ensured.

Psychological stress is a crucial area of research for psychological and neuroscientist. There have been many existing definitions of psychological stress which have culminated over the years by research done in the field and variations made in ICD and DSM manuals. A clever conceptualization of the same can be -

\section{"response to stressful stimuli"}

It is to be noted that perception of stressful stimuli is relative to each individual. While certain events due create a general level of stressful triggers like pandemics and terrorist attacks, the 
perception of individuals about such events and its impact on their lives contributes the most towards the existence of psychological stress triggers that they face. In context of COVID19 it means that, while an individual not diagnosed with COVID19 might be anxious and stressful regarding his susceptibility towards the disease, it is the more likely that the instilled uncertainty regarding his own future as a result of the world-level pandemic would be a major source of stress for him.

An acceptable approach towards understanding the psychological ripple of COVID19 on the minds of individuals in the Indian society, hence, would involve identifying possible triggers (or stressful stimuli) specific to individuals from each section of society which are most likely to induce a stressful response by the individual belonging to that particular section of society. To conduct a minimalistic study for the same, academic literature in the context of India primarily focused on studying impact of COVID19 on society was analyzed and studied to identify key notable triggers. The available databases of Google Scholar, PubMed, ProQuest, Scopus, Web of Science and PsyArxiV was used. The findings have been summarized in Table 1.

\section{Moving Beyond Existing Definition}

DSM-5 describes acute stress disorder as following -

"development of specific fear behaviors that last from 3 days to 1 month after a traumatic event."

While the definitions provided by manuals of DSM and ICD establish a basic starting point for understanding and diagnosing psychological stress among individuals, the possibility of such diagnosis becomes severely limited in times of public crisis events when masses of human population are most susceptible of being diagnosed of stress-related disorders. While few government agencies and NGOs come upfront post such crisis events to counsel the individuals directly affected by such events, a large scale counselling still couldn't be provided to those who are affected most by the ripple effect of these events. These are the ones indirectly affected by such events, but fail to receive requited counseling due to their absence in first-hand trauma of the public crisis events.

As a result, it becomes painfully essential that more insight shall be taken through the existing multi-modal techniques available that diagnose presence of psychological stress in the behaviors exhibited by the individual. Human behaviour, itself, is a multi-axial quantity that is manifested in varying proportions across the different axis of linguistic choices, psychological, emotional, acoustic variations, and even subtle implicit non-verbal cues. The observations that are gained from studying the impact of stressful events in general, and public crisis events in particular on these axes can help us establish more accurate and insightful diagnosis definitions for ICD and DSM. This shall also provide us great scale for building multi-modal stress detection systems.

Though, it is not possible to establish measures of mass-scale counselling, it is definitely possible to detect presence of psychological stress among these different axes of behaviour, which in turn can help us diagnose susceptibility to stress, or even stress (if multi-axial diagnosis is done) among individuals affected indirectly by such events. It should be noted that 
while DSM and ICD are essential part of the mental health literature, the obsessive reliance and addiction to view crisis events through the lenses of DSM and ICD leads to a blind spot towards the ripple effect victims of the events. As a result, they get neglected and often fail to receive any attention by researchers studying the impact of such events on society, and government agencies trying to identify groups of society to provide counselling measures post the occurrence of such events. [7].

\section{Methodology}

In our study, we shall aim our focus on detecting psychological stress among the acoustic axis for the group of migrant workers (more accurately known as daily-wage workers).

Due to existing lockdown measures, conducting field study would be a violation of government norms. As a result, the analysis of psychological stress along the acoustic variation axis becomes a more feasible and practical study to perform. The group of daily-wage workers have been taken due to the absence of literature for the same.

Our methodology shall be inspired from the works of Sondhi et al. 2015 [1] which aimed at identifying behavioral changes along the acoustic axis of the individual under psychological stress. Their study concluded the following two notable observations, which we aim to find in our study -

1. Mean fundamental frequency increases in speech of stressed individual

2. Distinct variation in spectrogram patterns of speech between stressed and non-stressed individual.

To obtain the acoustic samples of daily-wage workers across India, we shall be using publicly available TV interviews of daily-wage workers conducted by news broadcasting channel NDTV. [8]. These interviews were conducted on random dates starting from the month of March 2020 to May 2020 - the time period for implementation of strict lockdown and national curfew measures. Each interview conducted for a particular state contained conversational exchanges with more than ten daily-wage workers belonging to that state.

To count for representativeness of the study, we selected interviews of daily wage workers belonging from different states of India - Madhya Pradesh, Bihar and Delhi. The portions of the interviewer's voice were cut from the acoustic sample using tools of Audacity. For generation of spectrogram and mean frequency plots, the open-source tool named PRAAT was used [9-11].

To obtain speech of non-stressed individuals, random individuals were chosen through online volunteering forums who were provided the job for narration of the speech transcription of daily-wage workers. A total of ten random individuals were chosen from each of the states of Madhya Pradesh, Bihar and Delhi. A psychologist ensured that the chosen individuals were not suffering from any psychological stress that may affect the results of our study. At the end of the study, the random individuals were provided compensation for their volunteering work. The psychologist made sure that no cognitive bias could creep in during the period of study that might affect the results of the work.

\section{Inclusion Criteria for Control Group}


1. Individuals in the age group of 18-40. (Same age group as that of daily-wage workers interviewed).

2. Individuals not suffering from any psychological stress, or any psychological disorder.

3. Individuals belonging to same state as daily-wage workers. (Madhya Pradesh, Bihar and Delhi).

\section{Exclusion Criteria for Control Group}

1. Individuals susceptible to psychological stress.

\section{Sampling Size}

A sampling size of thirty individuals were chosen. Ten from each state.

After obtaining acoustic samples of stressed and non-stressed individuals, Audacity was used for normalization of samples. Finally, PRAAT was used to plot mean fundamental frequency plots and spectrograms for each sample.

\section{Results and Observations}

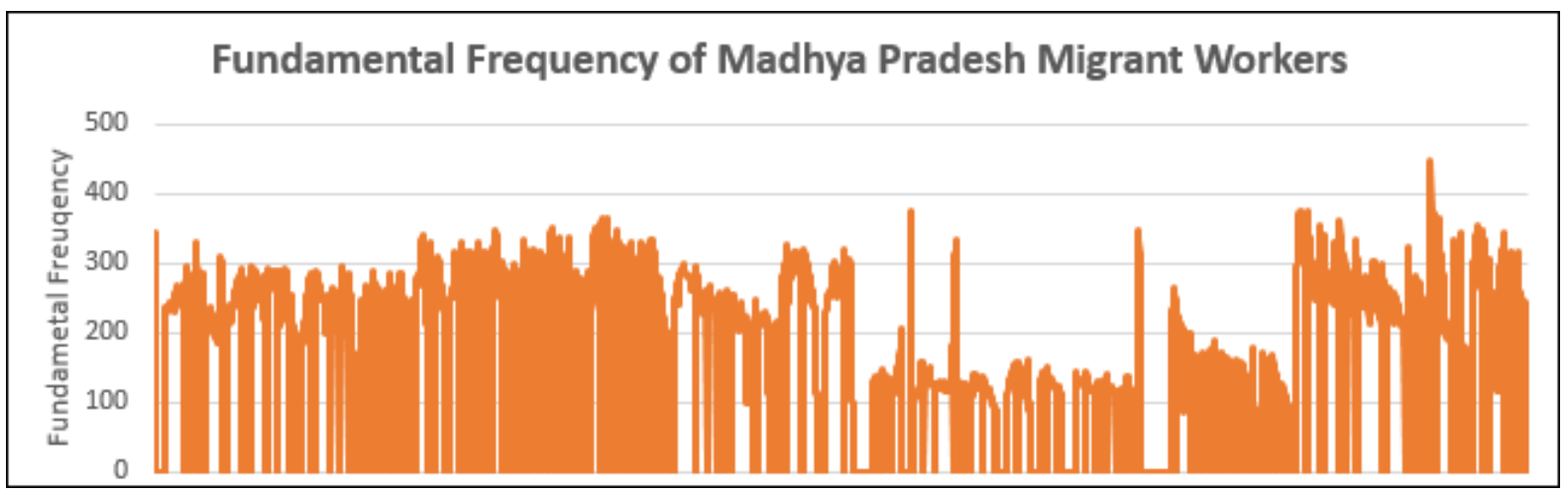

Figure 1. The above figure represents the fundamental frequency plot obtained from the acoustic samples of daily-wage workers from Madhya Pradesh

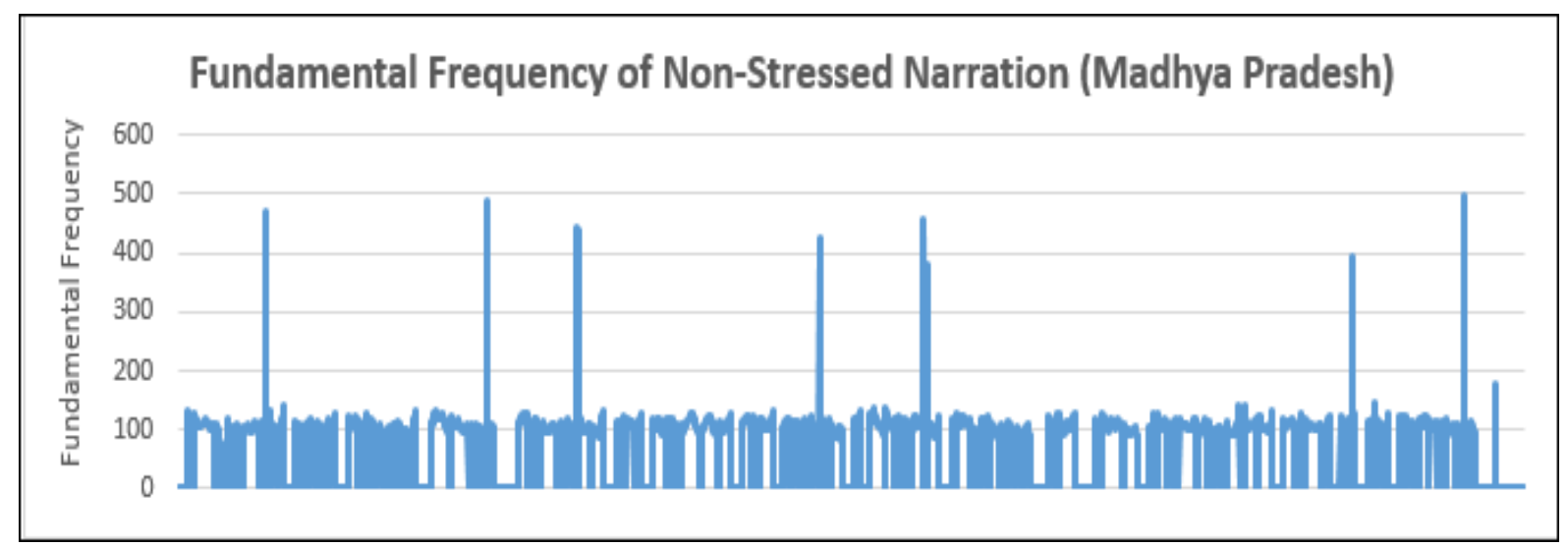

Figure 2. The above figure represents the fundamental frequency plot obtained from the acoustic samples from non-stressed narration of random individuals belonging to Madhya Pradesh 


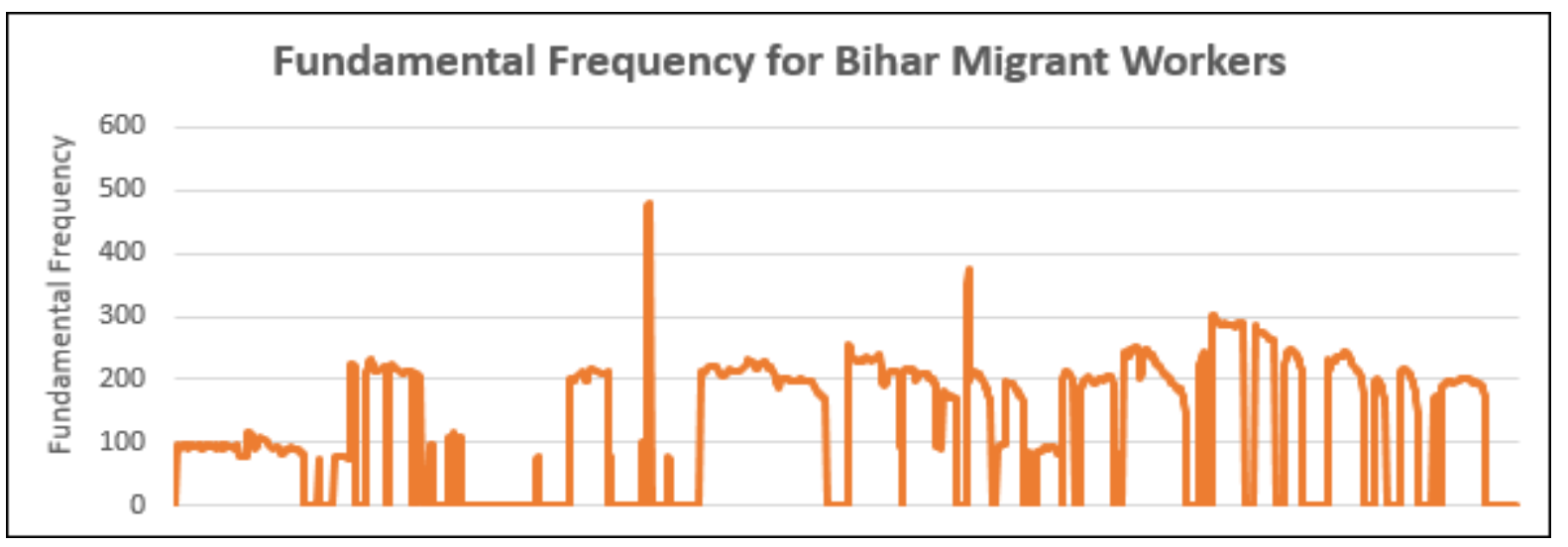

Figure 3. The above figure represents the fundamental frequency plot obtained from the acoustic samples of daily-wage workers from Bihar

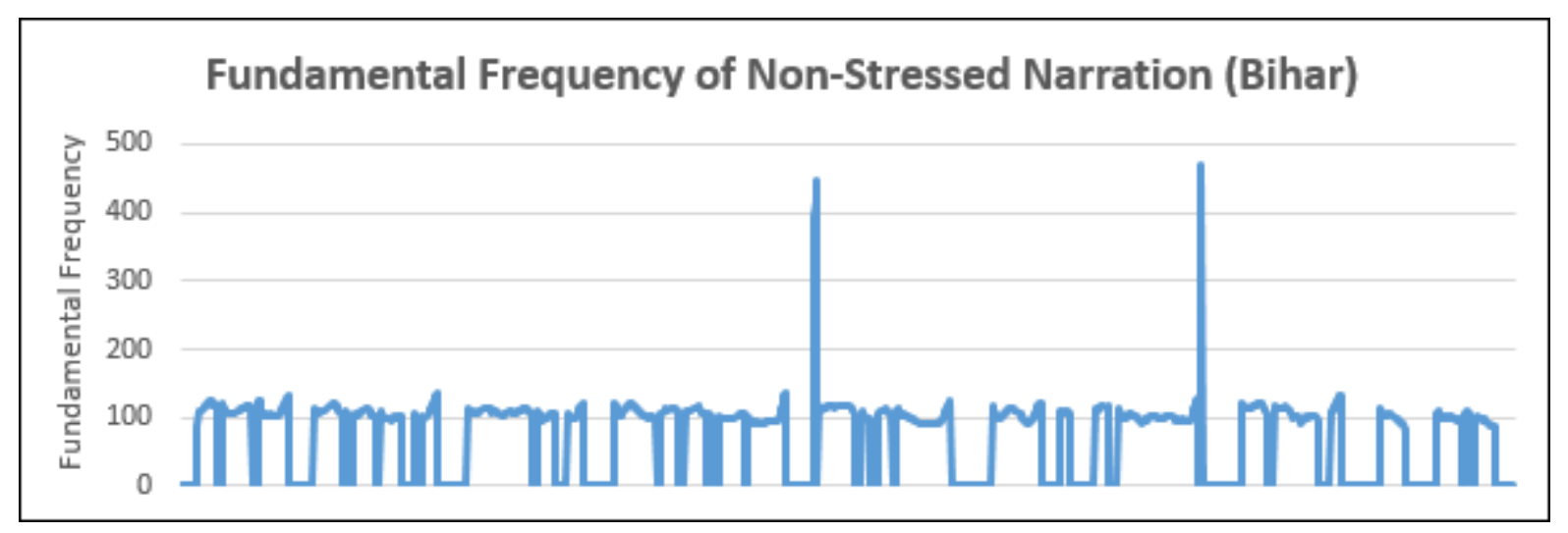

Figure 4. The above figure represents the fundamental frequency plot obtained from the acoustic samples from non-stressed narration of random individuals belonging to Bihar

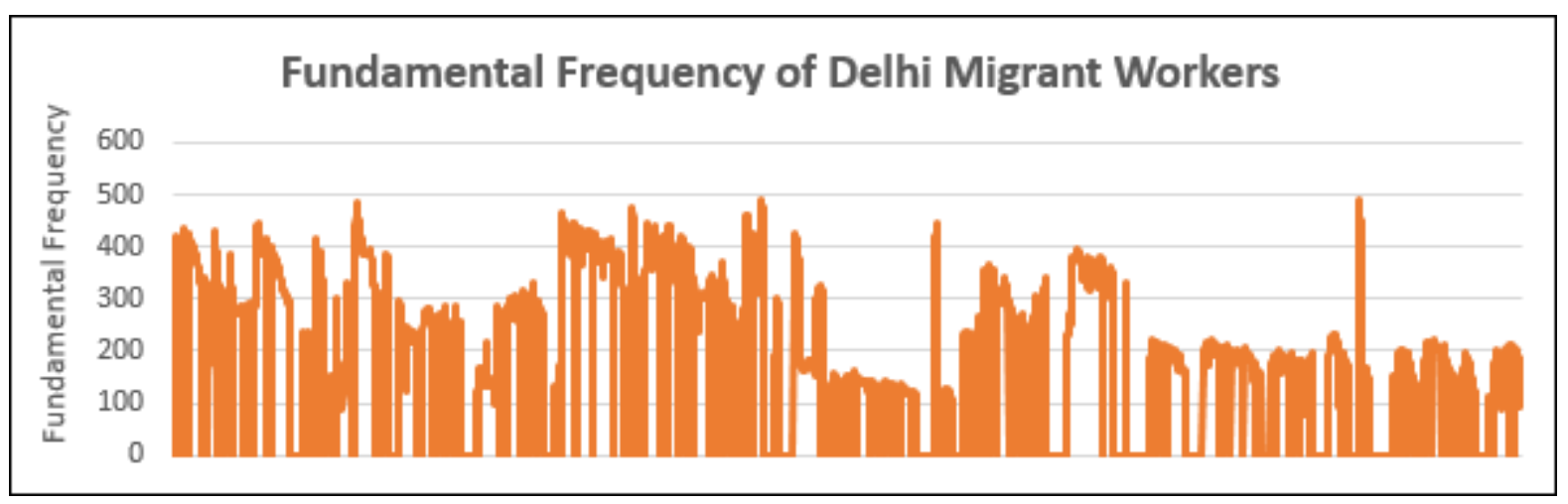

Figure 5. The above figure represents the fundamental frequency plot obtained from the acoustic samples of daily-wage workers from Delhi 


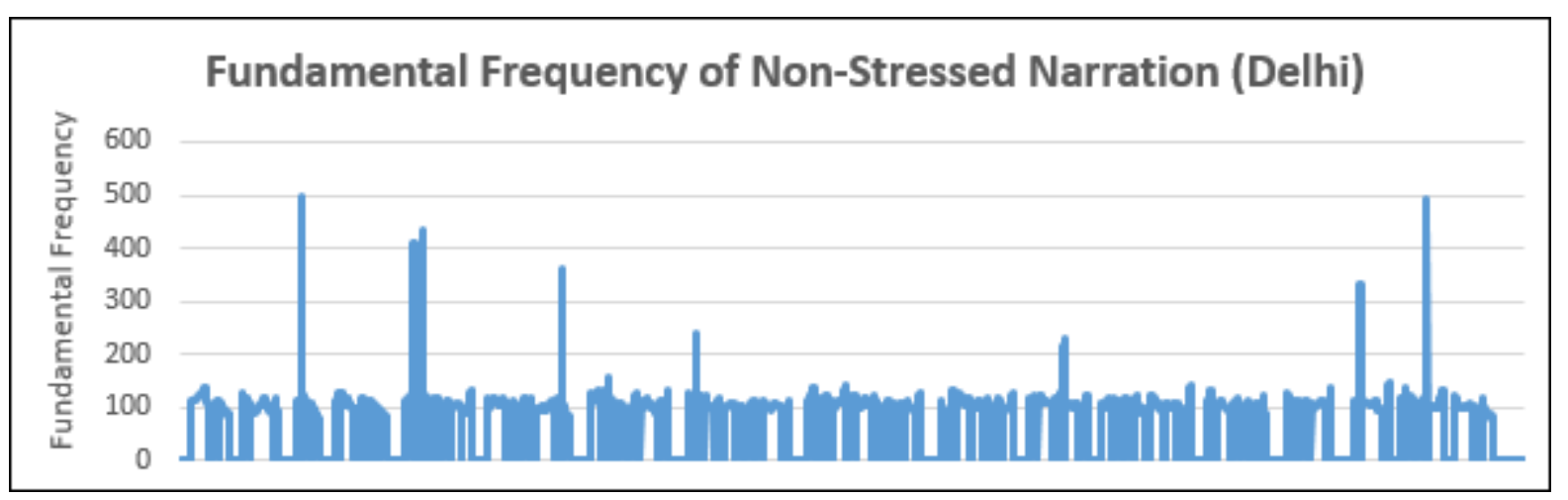

Figure 6. The above figure represents the fundamental frequency plot obtained from the acoustic samples from non-stressed narration of random individuals belonging to Delhi

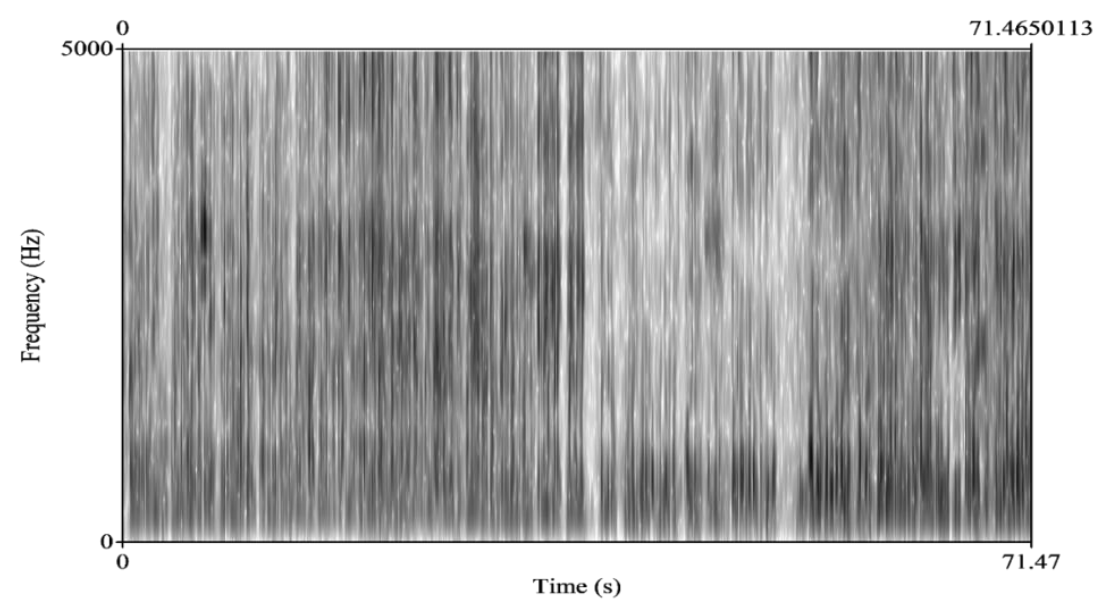

Figure 7. The above figure represents the spectrogram plot obtained from the acoustic samples of daily-wage workers from Madhya Pradesh

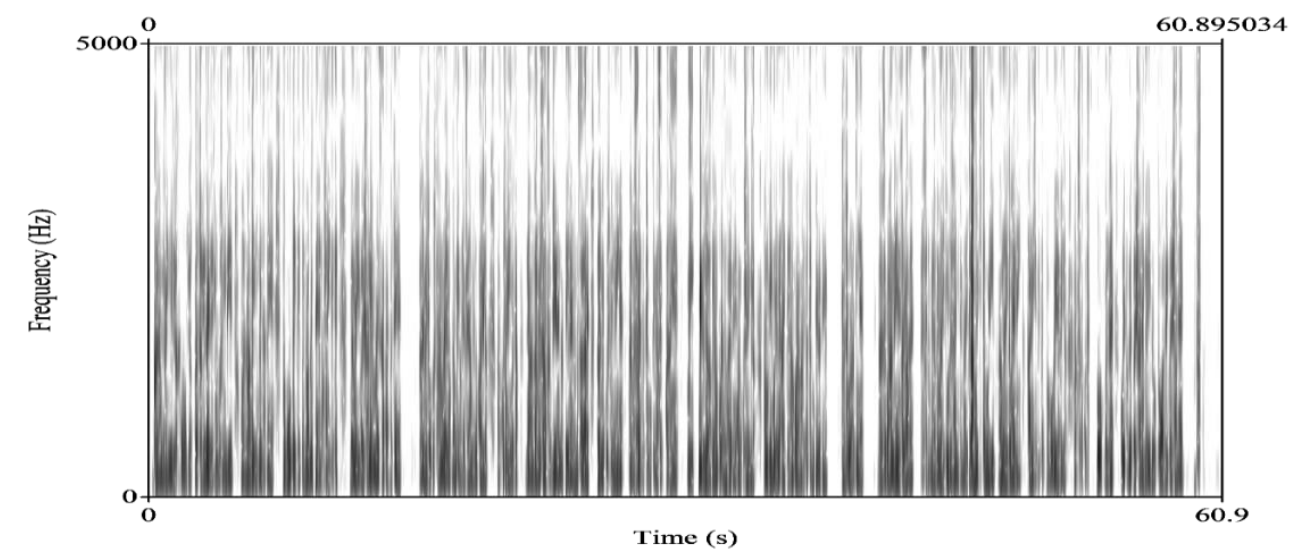

Figure 8. The above figure represents the spectrogram plot obtained from the acoustic samples from non-stressed narration of random individuals belonging to Madhya Pradesh 


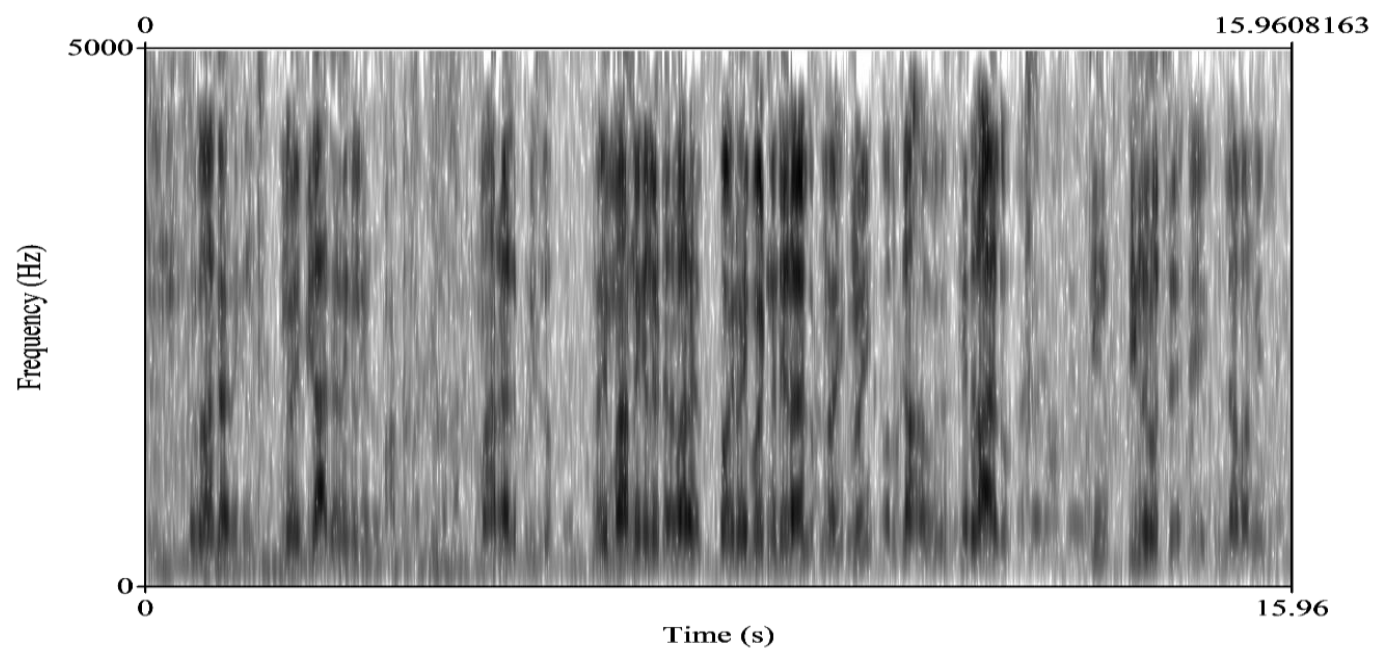

Figure 9. The above figure represents the spectrogram plot obtained from the acoustic samples of daily-wage workers from Bihar

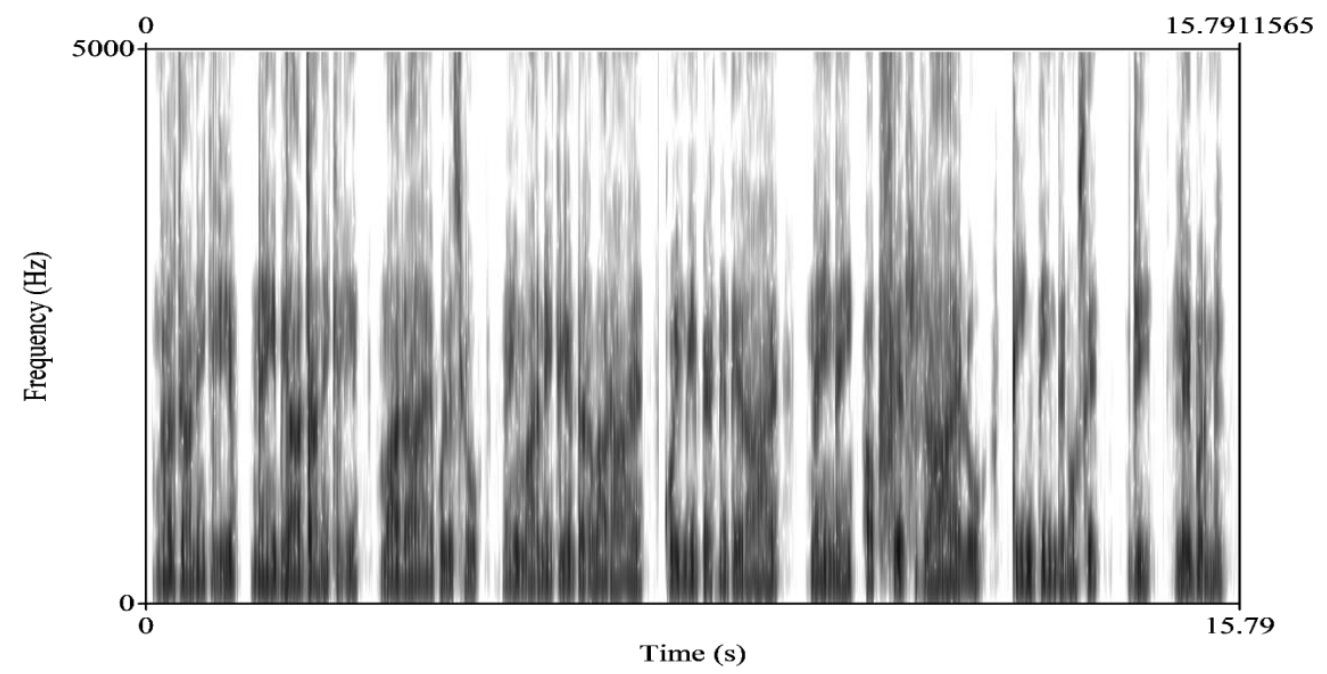

Figure 10. The above figure represents the spectrogram plot obtained from the acoustic samples from non-stressed narration of random individuals belonging to Bihar

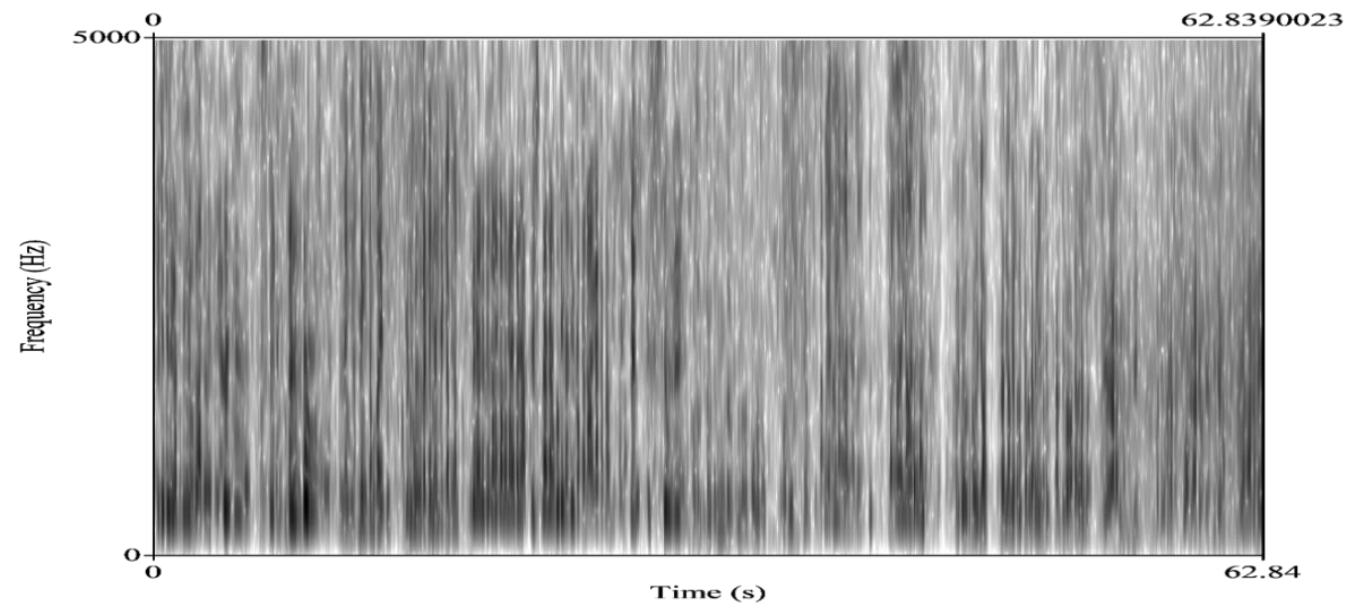

Figure 11. The above figure represents the spectrogram plot obtained from the acoustic samples of daily-wage workers from Bihar 


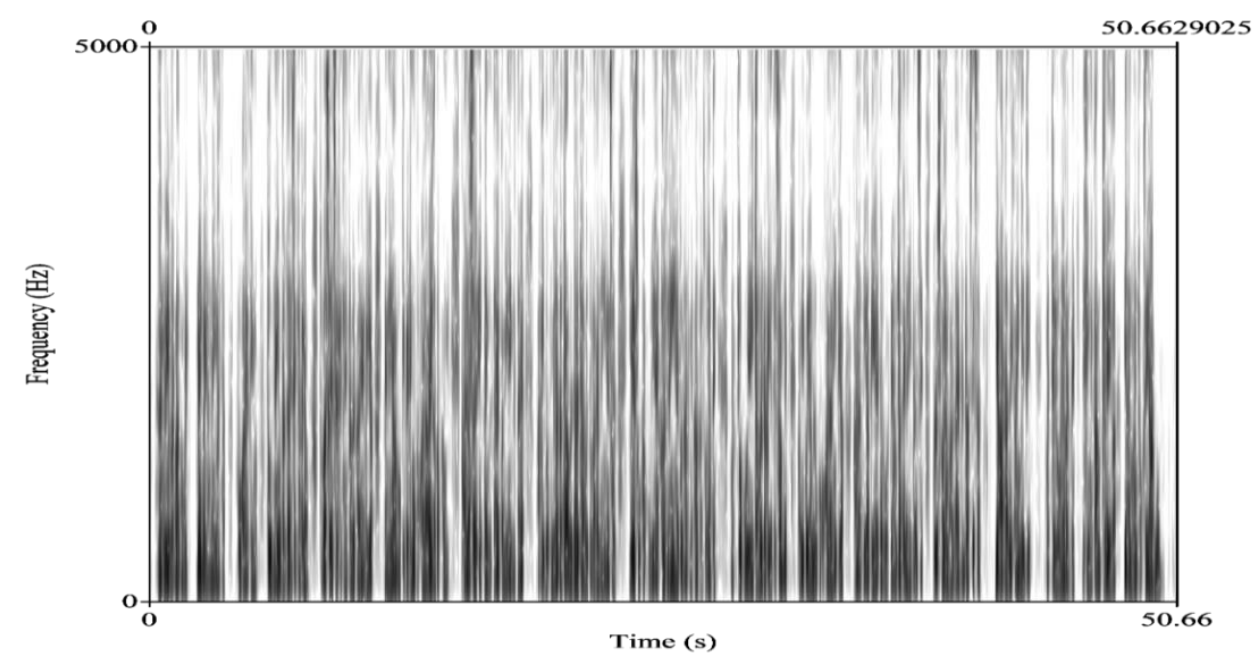

Figure 12. The above figure represents the spectrogram plot obtained from the acoustic samples from non-stressed narration of random individuals belonging to Delhi

The figures 1-12 summarize the results of our study. The figures 1-6 depict that stark differences in the fundamental frequency values of speech by stressed daily-wage workers and narrations by non-stressed individuals. The fundamental frequency plots of narrations by nonstressed individuals from each state are averaged to count for all ten random individuals chosen for each state. The figures 7-12 depict distinct density based variations in the spectrogram plots of acoustic samples for stressed daily-wage workers and narrations by non-stressed individuals. Like before, the plots obtained for non-stressed control group individuals are averaged to count for all the ten random individuals chosen for each state.

Our study, hence, concludes that along the acoustic variation axis of behavioral changes by individuals under psychological stress, migrant workers exhibit all the necessary signs identified by the works of Sondhi et al. in 2015. [1]. Hence, we can say, migrant workers are most susceptible of stress-related disorders, if diagnosed clinically. In either case, they exhibit signs of severe psychological stress, most likely due to stress triggers identified in Table 1.

\section{Suggestions}

The pandemic of COVID19 and the corresponding non-pharmaceutical interventions that have followed as its result has severely obstructed the conduction of psychological studies for groups affected by COVID19. In respect for the changing times and growing need for current psychological research, it becomes eminent to shift our attention towards remote/online studies that can utilize whatever data possible which can act as a representative sample of behavioral changes for the chosen group under study. [12-15].

Studies based on acoustic variational axis for other groups, linguistic axis for groups active on social media etc. can be conducted to identify possible susceptibility towards psychological stress. Results from remote/online based studies, then, can be used to counseling-inclusive non-pharmaceutical interventions by government agencies. 


\section{Statement regarding Authorship:}

The entire study was proposed, planned, and conducted solely, by the single author - Ajay Agarwal. No other person was involved in carrying out the study. The author shall be solely responsible for any questions that might arise.

\section{Disclosure of Any Potential Conflict of Interest}

None

Funding Sources (if any)

None

\section{References}

1. Sondhi S, Khan M, Vijay R, Salhan AK, Chouhan S. Acoustic analysis of speech under stress. IJBRA. 2015 Sep 1;11(5):417-32.

2. Chatterjee SS, Malathesh Barikar C, Mukherjee A. Impact of COVID-19 pandemic on pre-existing mental health problems. Asian Journal of Psychiatry. 2020 Jun;51:102071.

3. MacGeorge EL, Samter W, Feng B, Gillihan SJ, Graves AR. After 9/11: Goal disruption, emotional support, and psychological health in a lower exposure sample. Health Communication. 2007 Apr 10;21(1):11-22.

4. DiGrande L, Perrin MA, Thorpe LE, Thalji L, Murphy J, Wu D, Farfel M, Brackbill RM. Posttraumatic stress symptoms, PTSD, and risk factors among lower Manhattan residents 2-3 years after the September 11, 2001 terrorist attacks. Journal of Traumatic Stress: Official Publication of The International Society for Traumatic Stress Studies. 2008 Jun;21(3):264-73.

5. Taylor S, Landry C, Paluszek M, Fergus TA, McKay D, Asmundson GJ. Development and initial validation of the COVID Stress Scales. Journal of Anxiety Disorders. 2020 May 4:102232.

6. Sood S. Psychological effects of the Coronavirus disease-2019 pandemic. Research \& Humanities in Medical Education. 2020 Apr 1;7:23-6.

7. Bisson JI. What happened to harmonization of the PTSD diagnosis? The divergence of ICD11 and DSM5. Epidemiology and psychiatric sciences. 2013 Sep;22(3):205-7.

8. NDTV Interview Data.https://www.youtube.com/playlist?list=PLpSH-HCe7ZpZ81fUY2wg4BK7JWgmApeq\&advanced_settings=1\&disable_polymer=1

9. Styler, W. (2013). Using Praat for linguistic research. University of Colorado at Boulder Phonetics Lab

10. Owren, M. J. (2008). GSU Praat Tools: Scripts for modifying and analyzing sounds using Praat acoustics software. Behavior research methods, 40(3), 822-829

11. Amir, O., Wolf, M., \& Amir, N. (2009). A clinical comparison between two acoustic analysis softwares: MDVP and Praat. Biomedical Signal Processing and Control, 4(3), 202-205.

12. Das, N. (2020). Psychiatrist in post-COVID-19 era-Are we prepared?.Asian Journal of Psychiatry, 51, 102082-102082. 
13. Shevlin, M., McBride, O., Murphy, J., Miller, J. G., Hartman, T. K., Levita, L., ... \& Bennett, K. M. (2020). Anxiety, Depression, Traumatic Stress, and COVID-19 Related Anxiety in the UK General Population During the COVID-19 Pandemic.

14. Dutheil, F., Mondillon, L., \& Navel, V. (2020). PTSD as the second tsunami of the SARS-Cov2 pandemic. Psychological Medicine, 1-6.

15. Onyeaka, H. K., Zahid, S., \& Patel, R. S. (2020). The unaddressed behavioral health aspect during the coronavirus pandemic. Cureus, 12(3).

Tables

\begin{tabular}{|c|c|}
\hline Group of Individuals & $\begin{array}{c}\text { Stressful Stimuli/Triggers for } \\
\text { Psychological Stress }\end{array}$ \\
\hline Students & $\begin{array}{c}\text { Rescinding Job Offers, Degree Completion, } \\
\text { Uncertainty over Higher Education } \\
\text { Opportunities }\end{array}$ \\
\hline Migrant Workers & $\begin{array}{l}\text { Unemployment, Feeding their family, } \\
\text { Hunger, Money }\end{array}$ \\
\hline Corporate Employees & $\begin{array}{l}\text { Possibility of Losing Jobs, Feeding their } \\
\text { family }\end{array}$ \\
\hline Doctors and Nurses & $\begin{array}{l}\text { Curbing the Spread, Saving lives, Making } \\
\text { critical intubation decisions }\end{array}$ \\
\hline Teachers & $\begin{array}{l}\text { Feeding their family, Use of Technology for } \\
\text { Online Classes, Conduction of Exams }\end{array}$ \\
\hline Farmers & $\begin{array}{l}\text { Sale of Harvest, Sowing Crops for next } \\
\text { Harvest, Feeding their family }\end{array}$ \\
\hline $\begin{array}{c}\text { Police Officers and Law Enforcement } \\
\text { Agencies }\end{array}$ & $\begin{array}{l}\text { Maintaining Social Distancing among } \\
\text { public, Managing Containment Zones }\end{array}$ \\
\hline Shopkeepers and Grocery Stores & Sale of Products, Feeding their family \\
\hline $\begin{array}{c}\text { Cab Drivers and Public Transport } \\
\text { Drivers }\end{array}$ & Sale of Services, Feeding their family \\
\hline
\end{tabular}

Table 1. The above table lists the possible triggers of psychological stress for individuals belonging to different sections of the Indian Society 